\title{
Darmkrebs: Operation und Therapie bereits im frühen Stadium exakt planen - Ultraschall macht's möglich
}

Berlin - Tückisch, gefährlich und häufig: Darmkrebs verursacht im frühen Stadium oft keine Symptome, ist jedoch bei Frauen in Deutschland die zweithäufigste und bei Männern die dritthäufigste Tumorerkrankung. Die Endosonografie spielt bei den Enddarmtumoren eine äußert wichtige Rolle, um das Tumorstadium exakt einzugrenzen und damit die notwendige Therapie gezielt zu planen. Bisher gelingt es nur der Endosonografie, die Tumoreindringung in die Darmwand sicher zu bestimmen, darauf machen Experten der Deutschen Gesellschaft für Ultraschall in der Medizin e. V. (DEGUM) anlässlich des Darmkrebsmonats März aufmerksam. Die Ultraschalluntersuchung trägt entscheidend zur Planung einer Operation und zur Therapiewahl bei.

Wenn der Verdacht auf eine Darmkrebserkrankung besteht oder wenn sich Menschen bei einer Vorsorgeuntersuchung daraufhin untersuchen lassen, führen Ärzte in der Regel eine Darmspiegelung - also eine Endoskopie - durch. Dabei wird der Darm mit einem Endoskop von innen angeschaut. „Bei einer herkömmlichen Spiegelung des Darms sieht ein Arzt allerdings nur die Oberfläche der Darmwand“, sagt PD Dr. med. Michael Hocke, Leiter des DEGUM-Arbeitskreises Endosonografie. „Wenn die Untersuchung durch hochauflösende Ultraschallköpfe ergänzt wird, können hingegen genaue Schnittbilder der Darmwand gemacht werden." Bei der endoskopischen Ultraschalluntersuchung wird der Schallkopf - auch Sonde genannt - durch den Analkanal in den Körper eingeführt. So gelangt der Schallkopf besonders dicht an die zu untersuchenden Organe heran und kann fast mikroskopisch exakte Ultraschallbilder erzeugen, die besonders störungsfrei sind. „Der behandelnde Arzt kann dadurch sehr frühe Tumorstadien von bereits fortgeschrittenen exakt unterscheiden und beispielsweise erkennen, wie tief ein Tumor in die Darmwand eingewachsen ist und ob bereits benachbarte Organe oder Lymphknoten befallen sind“, so Hocke. Wenn der Tumor sich noch in einem sehr frühen Stadium befindet und beispielsweise noch nicht in die Darmwand eingewachsen ist, kann er unter Umständen direkt endoskopisch - also ohne eine Operation - entfernt werden. „Die endoskopische Ultraschalluntersuchung (EUS) hilft also stark bei der Entscheidung, ob eine Operation beim Enddarmtumor durchgeführt werden sollte oder nicht“, betont der DEGUM-Experte. „Zudem ermöglichen die detailgenauen sonografischen Bilder eine wertvolle Unterstützung bei der Planung der Operation, da sie das Darmgewebe sehr genau abbilden und so Auskunft darüber geben, ob vor der Operation eine Tumorverkleinerung durch eine Strahlen- und Chemotherapie erfolgen muss“, erwähnt Hocke.

Damit die Endosonografie-Diagnostik erfolgreich durchgeführt werden kann, ist es wichtig, dass der Untersucher erfahren ist. Die Endosonografie muss gezielt trainiert werden. Die DEGUM bietet dafür nach ihrem bewährten 3-Stufen-Konzept gezielte Fortbildungen für Mediziner an.

\section{HINTERGRUND: DARMKREBS}

Nach Angabe des Bundesministeriums für Gesundheit (BMG) erkranken in Deutschland jährlich mehr als 60000 Menschen an Darmkrebs. Im frühen Stadium treten oft keine oder nur unspezifische Beschwerden wie Verdauungsprobleme oder Blut im Stuhl auf. Vorsorgeuntersuchungen kommt deshalb eine besondere Bedeutung zu. Die rechtzeitige Erkennung kann die Heilungschancen bei Darmkrebs erheblich verbessern oder die Erkrankung sogar verhindern. Alle Krankenkassen übernehmen die Kosten für die Untersuchung zur Früherkennung von Darmkrebs für ihre Versicherten ab 50 Jahren. Im Rahmen der Vorsorgeuntersuchung haben die Patienten im Alter von 50 bis 54 Jahren die Möglichkeit, einen jährlichen Stuhltest machen zu lassen. Eine Darmspiegelung wird Männern ab 50 und Frauen ab 55 Jahren zudem empfohlen und von den Krankenkassen finanziert. Wenn die Erkrankung bereits in der Familie aufgetreten ist, wird die Vorsorgeuntersuchung frühzeitiger von den Krankenkassen übernommen. 